\title{
An unusual case of ovarian torsion with infarction simulating malignancy in an adolescent girl
}

\author{
Madhu Jain ${ }^{1 *}$, Kusum Lata ${ }^{1}$, Shivi Jain ${ }^{2}$ \\ ${ }^{1}$ Department of Obstetrics \& Gynecology, Institute of Medical Sciences, Banaras Hindu University, Varanasi, India; \\ ${ }^{2}$ Department of Obstetrics \& Gynecology and Radiology, Institute of Medical Sciences, Banaras Hindu University, Varanasi, India \\ E-mail: *drmadhujainbhu@gmail.com
}

Received 11 June 2011; revised 20 August 2011; accepted 27 August 2011.

\begin{abstract}
Torsion of the ovary is an uncommon condition. It is the $5^{\text {th }}$ most common gynaecological emergency. It most commonly occur in reproductive age group, on right side, often in presence of congenital abnormalities of adnexa but may occur in their absence too. Adnexal torsion often poses a diagnostic challenge because of non specific symptoms of presentation. Ultrasound with colour Doppler may be helpful.
\end{abstract}

Keywords: Adenexal Torsion; Ovarian Infarction; Colour Doppler

\section{INTRODUCTION}

Torsion of uterine adnexa is an uncommon condition. Torsion of ovary is still uncommon. Despite of being uncommon this is the $5^{\text {th }}$ most common gynaecological emergency [1], representing 3\% of the emergency gynaecological surgeries [2]. Adnexal torsion is a diagnostic dilemma largely because of the non specific clinical, laboratory, and imaging findings [3].

\section{CASE REPORT}

A 15 year old unmarried girl Miss M.R., presented in our out patient department with the complains of dull aching pain in lower abdomen for one year. It was more on right side, episodic, non radiating, not responding to medications. The pain was increased in severity for last five to six days. It was constant, radiating to suprapubic region, with some relief on medication not associated with nausea or vomiting. For this ultrasound was done in a private hospital and told of having some tumour. She denied of having any gastrointestinal or genitourinary symptoms. She achieved menarche one year back. Her cycles were irregular at 2 - 3 months, of 8 - 10 days duration with average flow. Her last menstrual period was seven days back.

On examination she was alert, oriented and in severe agony due to the pain. Her temperature was normal, respiratory rate was 16 breaths/min, heart rate was 88 beats/min, and blood pressure was 110/70 mm Hg. Her secondary sexual characters were normally developed. Her cardiovascular and respiratory examinations were unremarkable. Abdominal examination revealed a firm lump in the lower abdomen of approximately 24 weeks size involving iliac fosae, both lumbar, umbilical and hypogastric areas. It was immobile, tender, overlying skin was normal looking, lower margins were not reached. She had no hepatosplenomegaly and bowel sounds were normal. In per rectal examination, rectal mucosa was free and fullness was felt anteriorly.

A pelvic ultrasound with colour Doppler was performed, showing a large heterogeneous well defined elongated pelvic mass of $17.3 \times 11.1 \times 9 \mathrm{~cm}$, arising from right adnexa with multiple cystic areas and right sided subhepatic extension, suggestive of ovarian mass (Figure 1). There was no significant colour Doppler flow. Both ovaries were not visualized separately. How- ever CECT pelvis revealed heterodense solid cystic mass lesion in pelvis arising from right adnexal region with mild pelvic ascites and mesenteric fat stranding, enlarged pelvic lymph node, suggestive of malignant right ovarian mass (Figure 2(a), 2(b)). MRI also reported a well defined predominantly cystic mass and an eccentric solid component, with an extension and encasement of right distal common iliac, proximal external iliac and infiltration of neurovascular bundle, suggestive of Germ cell tumour with possibility of malignant degeneration (Figure 3(a), 3(b)). Patient was also evaluated with tumour markers such as LDH, $\beta$-HCG, Ca-125 \& $\alpha$ fetoprotein, out of which only LDH was mildly increased. Laparotomy was done after proper evaluation. A large blackish right ovarian mass of approximately $25 \times$ $15 \mathrm{~cm}$ was present (Figure 4). On delivering the ovary out, 4 - 5 twist was present around ovarian ligament. Histopathology turned out to, the right ovarian haemorrhagic cyst. 


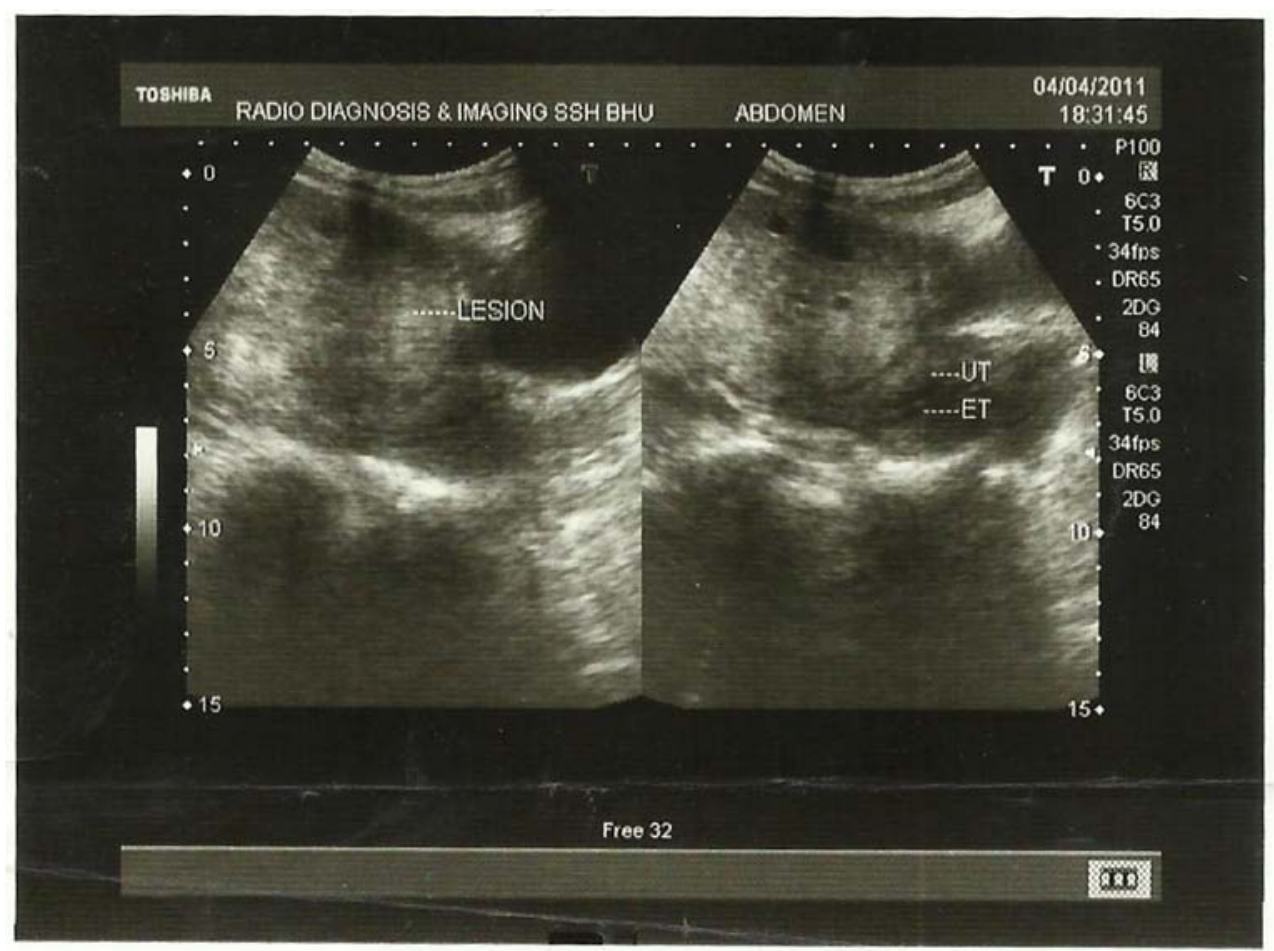

Figure 1. A large heterogeneous well defined mass with multiple cystic areas in USG.
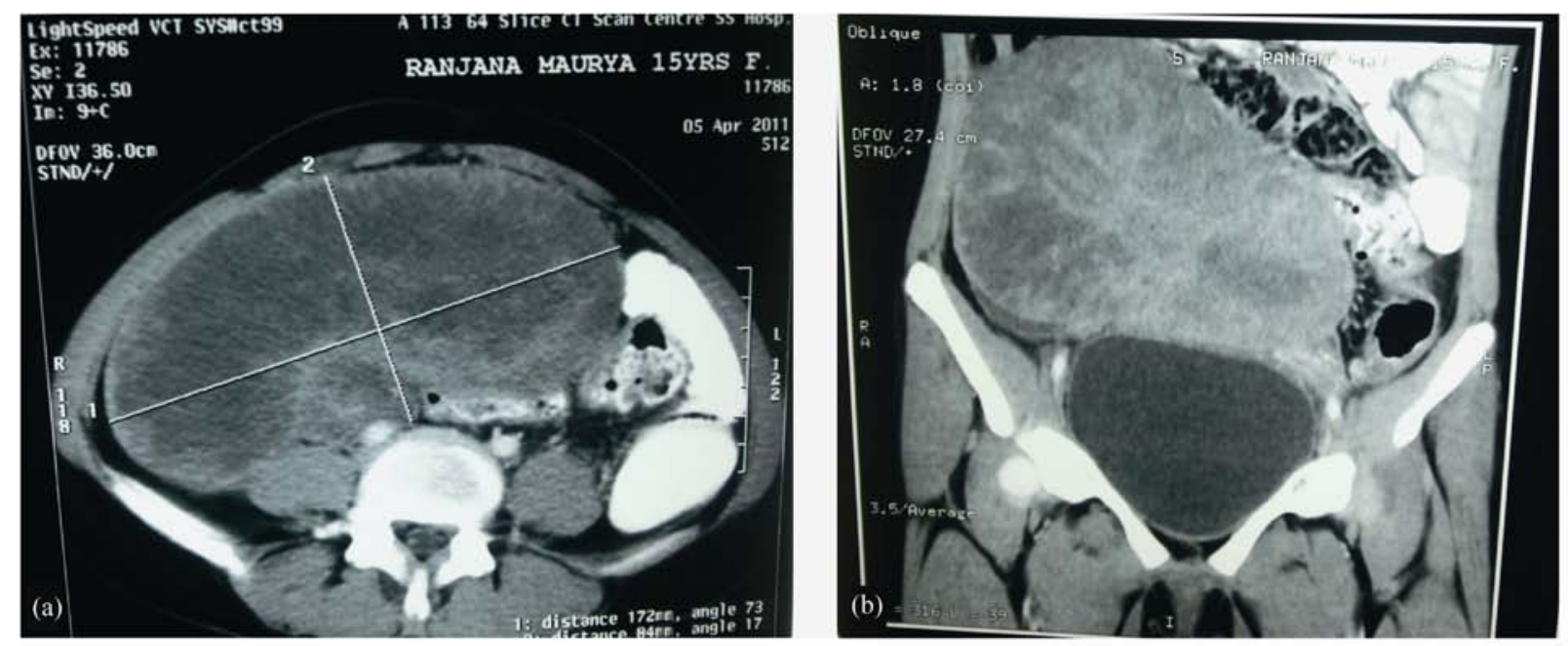

Figure 2. CECT Pelvis. (a) Axial plane shows a well defined heterodense solid cystic mass lesion $(17.2 \times 8.4 \mathrm{~cm})$ arising from right adnexal region and encasing right distal common iliac artery. (b) Coronal plane shows extension of right adnexal mass into hypogastrium, right iliac fossa, lumbar and epigastrium causing displacement of surrounding bowel loops.

\section{DISCUSSION}

Ovarian torsion first described by Kuestner in $1891^{1}$, results from partial or complete rotation of the ovarian pedicle on its long axis, potentially compromising venous and lymphatic drainage. If the rotation is partial or intermittent, the venous and lymphatic congestion may subside quickly, along with symptoms. If rotation is complete and prolonged, venous and arterial thrombosis occur, ultimately causing infarction. Volume of the twisted ovary averages 28 times the normal size. The ovarian stroma may be heterogeneous due to haemor-rhage and oedema. Torsion is rarely bilateral and is more common on the right side, perhaps because the sigmoid colon leaves limited space for left adnexal mobility. Torsion occurs more commonly in young women, with the greatest incidence in the 20 to 30 year age group [1]. 

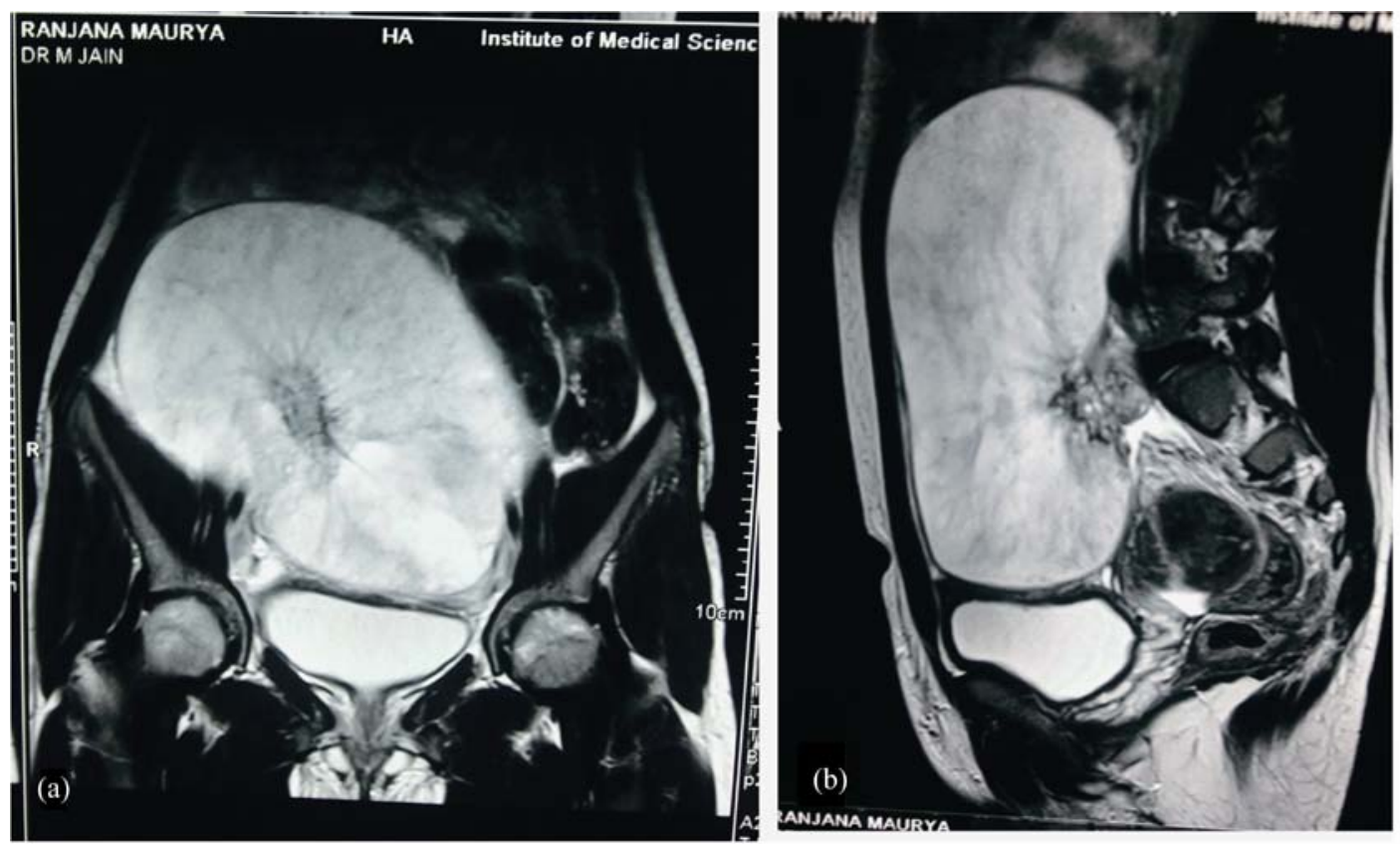

Figure 3. MRI Pelvis of the same patient. (a) Coronal plane in $\mathrm{T}_{2} \mathrm{~W}$ sequence shows a well defined right adnexal mass with an eccentric solid mural nodule. (b) Sagittal plane in $\mathrm{T}_{2} \mathrm{~W}$ sequence shows superior extension of the mass into lower abdominal cavity with an eccentric solid component in the posterior wall.
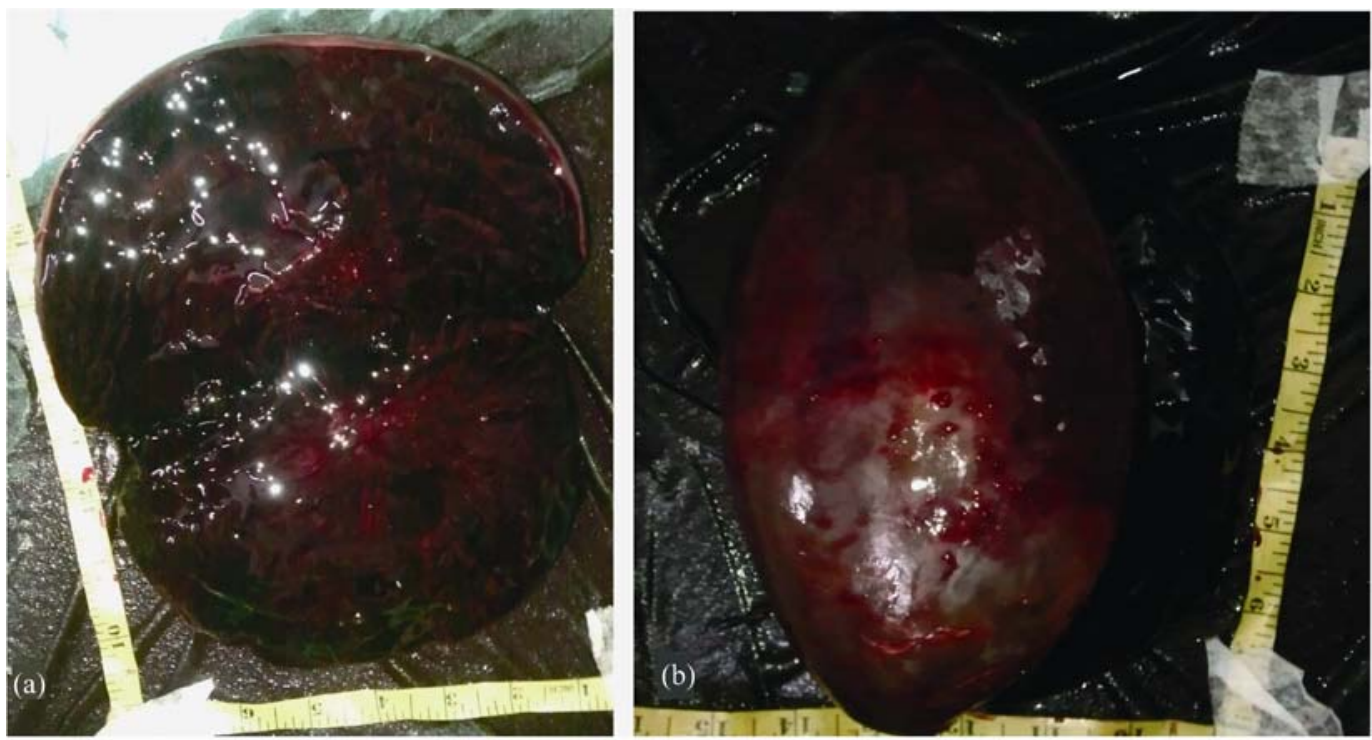

Figure 4. A large, blackish, infarcted right ovarian mass.

Torsion of a normal ovary is unusual but is more common in adolescents in whom developmental abnormalities are there such as markedly mobile fallopian tubes, absent mesosalpinx, elongated pelvic ligaments. Fallopian tube spasm, strenuous exercise or abrupt changes in intraabdominal pressure can also cause torsion of normal ovaries. These factors might be responsible for torsion in our patient in the absence of congenital abnormality. The varied imaging features and nonspe- cific symptoms of ovarian torsion can lead to a delay in identification with misdiagnosis being common [4].

\section{CONCLUSIONS}

Ovarian torsion is a surgical emergency that often presents a difficult diagnostic challenge. A detailed history and physical examination including a pelvic examination is necessary. Ancillary tests such as tumour markers are useful to exclude the possibilities of malignancy. Ultra- 
sound with or without colour Doppler imaging may be helpful.

\section{REFERENCES}

[1] Hibbard, L.T. (1985) Adnexal torsion. American Journal of Obstetrics and Gynecology, 152, 456-461.

[2] Chris, M. and Kiek, M. (2006) Ovarian torsion in a 20 year old patient. Canadian Journal of Emergency Medicine, 8, 126-129.

[3] Mark, A.H. and Terry, M.S. (1989) Ovarian torsion: Sonographic evaluation. Journal of Clinical Ultrasound, 17, 327-332.

[4] Hannah, C.C., et al. (2008) Pearls and pitfalls in diagnosis of ovarian torsion. Radiographics, 28, 1355-1368. doi:10.1148/rg.285075130 